\title{
Enhanced Magnetoresistive Effect in the Arrays of Nickel Nanorods on Silicon Substrates
}

\author{
Yu. A. Fedotova ${ }^{a}$, D. K. Ivanov ${ }^{b}$, Yu. A. Ivanova ${ }^{b}$, A. Saad ${ }^{c}$, A. V. Mazanik ${ }^{b}$, I. A. Svito ${ }^{b}$, \\ E. A. Strel'tsov ${ }^{b}$, A. K. Fedotov ${ }^{b}$, S. I. Tyutyunnikov' ${ }^{d}$, and P. Yu. Apel'd \\ ${ }^{a}$ National Center of Physics of Particles and High Energies, Belarussian State University, Republic of Belarus \\ ${ }^{b}$ Belarussian State University, Republic of Belarus \\ ${ }^{c}$ Al-Balqa Applied University, Jordan \\ ${ }^{d}$ Joint Institute of Nuclear Research, Russia \\ e-mail:julia@hep.by \\ Received July 25, 2012
}

\begin{abstract}
It is shown that the magnetoresistive properties of $n-\mathrm{Si} / \mathrm{SiO}_{2} / \mathrm{Ni}$, nanostructures containing nanogranular nickel rods in $\mathrm{SiO}_{2}$ layer's vertical pores substantially differ from the similar properties in the earlier studied nanogranular Ni films electrodeposited onto the $n-\mathrm{Si}$ plates. From the point of view of the electrophysical properties, the nanostructures studied are analogous to a system of two Schottky $\mathrm{Si} / \mathrm{Ni}$ diodes, which are connected to each other. The magnetoresistance of such structures has been studied in the temperature range from 2 to $300 \mathrm{~K}$ and the magnetic field range of up to $8 \mathrm{Tl}$. It is established that at temperatures of $17-27 \mathrm{~K}$ the structures possess a positive magnetoresistive effect, whose value depends on the transverse voltage applied to the structure and increases with a decrease in the longitudinal (along the rods) current intensity. At a current of $100 \mathrm{nA}$, the relative magnetoresistance in the field of $8 \mathrm{Tl}$ increases from 500 to $35000 \%$ by an increase in the transverse voltage from 0 to $-2 \mathrm{~V}$. The observed magnetoresistive effect is associated with the influence of the magnetic field on the processes of impact ionization of impurities resulting in an avalanche breakdown of the $\mathrm{Ni} / \mathrm{Si}$ Schottky barrier. Thus, the possibility of controlling the magnetoresistive effect in $n-\mathrm{Si} / \mathrm{SiO}_{2} / \mathrm{Ni}$ template structures by applying an additional (transverse) electric field to the nanostructure between the silicon substrate (as the third electrode) and nickel rods is proven.
\end{abstract}

Keywords: Schottky barrier, $\mathrm{Si}$, magnetoresistance, magnetoresistive effect, nanostructures, $\mathrm{Ni}$

DOI: $10.1134 / \mathrm{S} 1063739715080053$

\section{INTRODUCTION}

The existing magnetic nanosensors and memory cells possess a number of disadvantages associated with the strong sensitivity to temperature, high cost, and degradation of the characteristics at high frequencies. In this respect, the development of methods for synthesizing nanostructures and their arrays, which use the effects of giant or tunnel magnetoresistance, is of interest [1]. The effects indicated are implemented in layered systems containing a large number of magnetic and nonmagnetic layers forming superlattice [2], multilayered structures in a configuration of spin gates [3], contacts between two ferromagnetic materials [4, 5], etc.

Another approach to the creation of the magnetic sensor's arrays is based on the application of porous matrices (templates) $\left(\mathrm{SiO}_{2}, \mathrm{Al}_{2} \mathrm{O}_{3}\right.$, polymers, etc.), in which ordered or randomly distributed mesa- or nanopores may be filled with various materials [6]. Template synthesis has become an available and reliable method for creating nanoparticle arrays of various metal and semiconductor materials (see, e.g., [1, 7, $8]$ ). One of the template synthesis methods is based on the application of ion tracks. It is known that an irradiation with heavy high-energy ions can substantially change the properties of the material in a cylindrical region of a diameter of up to $10 \mathrm{~nm}$. A selective etching of these latent ion tracks, which results in the creation of channels (pores) with a large aspect ratio length/diameter, makes it possible to form porous templates for the subsequent formation of an array of nanorods.

The application of the template synthesis is of special interest for the development of micro- and nanoelectronics devices in combination with the electrochemical deposition, which makes it possible to control the growth processes on an atomic level. The advantage of the electrochemical deposition is the efficient control of the thickness, composition, and size of the sediment grains. The variation in the synthesis parameters (the potential of deposition, electrolyte's composition, temperature, duration) makes it possible to create both homogeneous materials and heterogeneous structures [9].

The considered method of synthesizing a nanoparticle's arrays on silicon substrates, which reveal their 
magnetoresistive properties, is promising for the development of magnetosensitive electronic devices compatible with the planar silicon technology. Besides, by the creation of a nanoparticle's arrays on a semiconductor substrate, the latter can be used as an additional electrode. As a result, the possibility arises of controlling the parameters of the obtained structures by varying the applied electric voltage $[10,11]$.

This work is aimed at studying the magnetoresistive properties of $n-\mathrm{Si} / \mathrm{SiO}_{2} / \mathrm{Ni}$ nanostructures, in which nickel is electrochemically deposited into the nanopores created by the selective etching of tracks of heavy ions in a $\mathrm{SiO}_{2}$ layer, which was thermally grown on a silicon substrate.

\section{EXPERIMENTAL}

A layer of $\mathrm{SiO}_{2}$ with a thickness of $700 \mathrm{~nm}$ was obtained by the thermal oxidation $\left(1100^{\circ} \mathrm{C}, 10 \mathrm{~h}\right.$, pure oxygen) of silicon wafers of brand KEF 4.5 (surface orientation (100)). Then, the wafers were irradiated with ${ }^{197} \mathrm{Au}^{26+}$ ions with an energy of $350 \mathrm{MeV}$ and a fluence of $5 \times 10^{8} \mathrm{~cm}^{-2}$. The pores were obtained by selective chemical etching of the latent ion tracks in a weak solution of hydrofluoric acid. The etching resulted in the formation of a system of randomly located through nanopores over the whole silicon oxide's thickness. The pores had a shape of a truncated cone with lower and upper diameters of the order of 100 and $250 \mathrm{~nm}$, respectively. The nanopores were filled with nickel by electrochemical deposition in an underpotential mode. The modes of deposition and the electrochemical properties of the obtained nanostructures are described in [12].

The obtained $n-\mathrm{Si} / \mathrm{SiO}_{2} / \mathrm{Ni}$ nanostructures were studied by scanning electron microscopy (SEM) and $\mathrm{X}$-ray phase analysis (XPA). According to [12], the electrodeposited nickel possesses a face-centered cubic latitude ( $a \approx 0.352 \mathrm{~nm}$ ) and a granular structure with the size of granules of the order of 30-70 nm.

The measurements of the electrophysical properties were carried out in a temperature interval from 2 to $310 \mathrm{~K}$ in magnetic fields up to $8 \mathrm{Tl}$ with the use of a system CFM (Cryogenic Ltd., London) based on a refrigerator with a closed cycle. The temperature controller used (Lakeshore, model 331) allowed us to implement a continuous sweeping of the temperature at a rate of $0.1-1 \mathrm{~K} / \mathrm{min}$ or to stabilize the temperature with an accuracy of $0.005 \mathrm{~K}$ in the process of magnetic field sweeping or measurements of voltage-current characteristics [12].

The voltage-current characteristics of the studied $n-\mathrm{Si} / \mathrm{SiO}_{2} / \mathrm{Ni}$ nanostructures (Fig. 1a) were measured with the use of three contacts (Fig. 1b). Contacts 1 and 2 were formed at the sample's face surface using an ultrasound soldering of indium. The rear contact 3 was formed on the back surface of the silicon substrate using indium-gallium eutectics. The presence of a

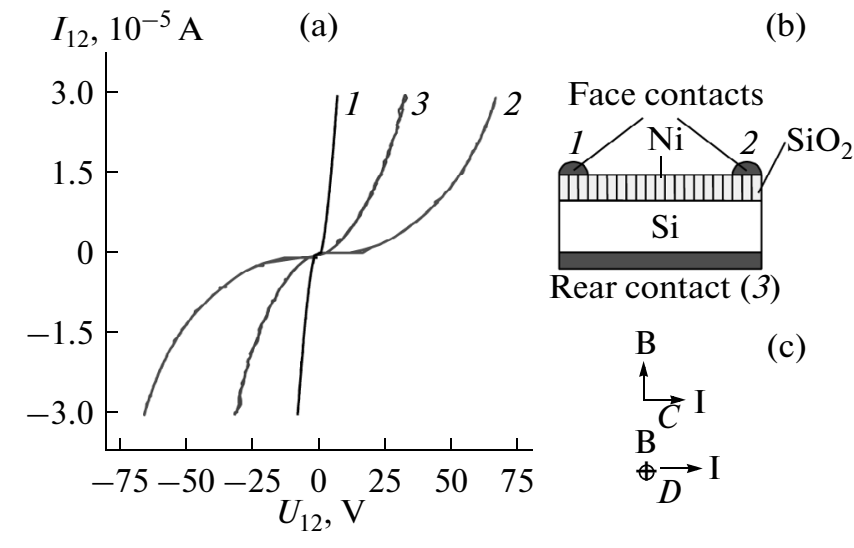

Fig. 1. Voltage-current characteristics of $n-\mathrm{Si} / \mathrm{SiO}_{2} / \mathrm{Ni}$ nanostructures at $V_{\mathrm{tr}}=0$ and $T=25 \mathrm{~K}$ (a), location of the contacts (b), and the relative directions of the magnetic field and current for configurations $C$ and $D$ (c): (a): (1) $B=0$; (2) $B=8 \mathrm{Tl}$, configuration $C$; (3) $B=8 \mathrm{Tl}$, configuration $D$.

rear contact allowed us to apply a transverse voltage $V_{\text {tr }}$ to the structure (between the pairs of contacts 1 and 3 or 2 and 3 ).

The resistance between contacts 1 and 2 was determined as the ratio of the voltage between these contacts $V_{12}$ to current $I_{12}$. By measuring the temperature dependence of $R_{12}$, the temperature was decreased from room temperature to $2 \mathrm{~K}$ at a rate of $0.5 \mathrm{~K} / \mathrm{min}$ and current $I_{12}$ was kept constant. As can be seen from Fig. 1b, by such a scheme of measurements, current $I_{12}$ always went through the bonds of the nickel rods under contacts 1 and 2 and then flowed along the substrate in parallel to the sample's surface.

The relative magnetoresistance was determined according to the relation

$$
M R_{12}(B)=\frac{R_{12}(B)-R_{12}(0)}{R_{12}(0)}=\frac{V_{12}(B)-V_{12}(0)}{V_{12}(0)},
$$

where $R_{12}(B)$ and $R_{12}(0)$ are the resistances of the nanostructure in magnetic field $B$ and in its absence, respectively. The value of $M R_{12}$ was calculated by subtracting from each other the temperature dependences of $R_{12}(T)$, which were measured at $B=0$ and $B=8 \mathrm{Tl}$.

The magnetoresistance was measured at two different mutual orientations of the magnetic field and current $I_{12}$ through the sample (configurations $C$ and $D$, Fig. 1c). As can be seen from Fig. 1c, in configuration $C$, vector $\mathbf{B}$ is directed along the axis of the nickel nanorods, while in configuration $D$, it is perpendicular both to the axis of the nanorods and the direction of the current.

\section{RESULTS AND DISCUSSION}

Figure 1 shows an example of the voltage-current characteristics for the nanostructures studied, which 


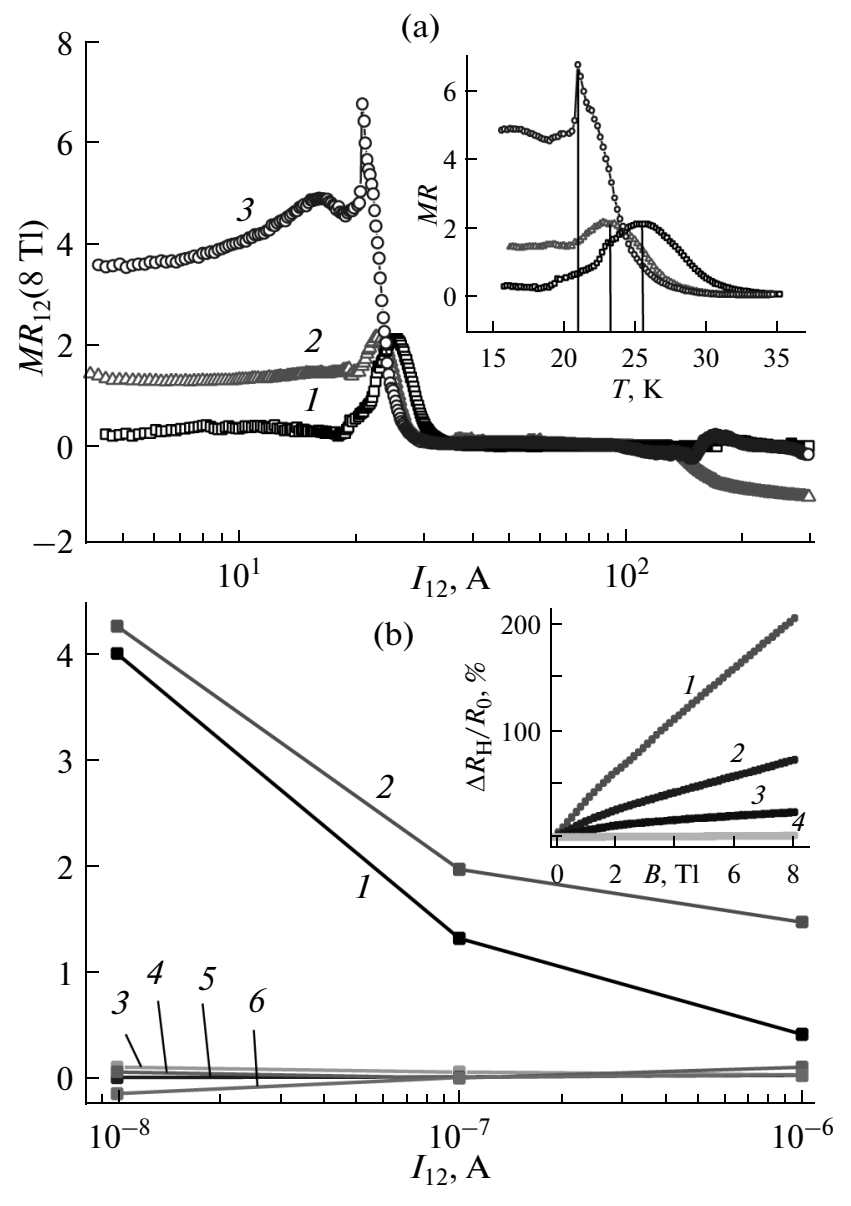

Fig. 2. Dependences of the magnetoresistance $M R_{12}$ for the geometry of measurement $C$ on temperature $T$ (a) and current strength $I_{12}$ (b) at $V_{\mathrm{tr}}=0$ and $B=8 \mathrm{Tl}$ : (a): (1-3) for current $I_{12} 1000,100$, and $10 \mathrm{nA}$, respectively; (b): (1-6) at a temperature of $10,23,50,100,160,300 \mathrm{~K}$. Insert in Fig. $2 \mathrm{a}$ is the shift in the maximum of the magnetoresistance with a decrease in current $I_{12}$; insert in Fig. $2 \mathrm{~b}$ are the magnetofield dependences $M R_{12}(B)$ measured at $I_{12}=100 \mathrm{nA}$ in the configuration $C$ at $T=23$ (1), 25 (2), $30(3)$, and $50 \mathrm{~K}(4)$.

were measured at $25 \mathrm{~K}$ in the absence of the transverse bias voltage. The symmetry of the $I-V$ branches corresponding to different polarities of the longitudinal voltage $V_{12}$ indicates that the bonds of nickel nanorods located under contacts 1 and 2 (Fig. 1, see Insert (i)) form two systems connected in series through the substrate and the Schottky $\mathrm{Si} / \mathrm{Ni}$ diodes enabled towards each other. As can be seen from Fig. 1, the enabling of a magnetic field causes a decrease in the current, and the value of this effect depends on the orientation of the vector B with respect to the substrate's plane and nickel nanorods. This is in agreement with the previous work [12], where the presence of a substantial positive magnetoresistive effect in the temperature range of $17-27 \mathrm{~K}$ was first observed for $n-\mathrm{Si} / \mathrm{SiO}_{2} / \mathrm{Ni}$ nanostructures.

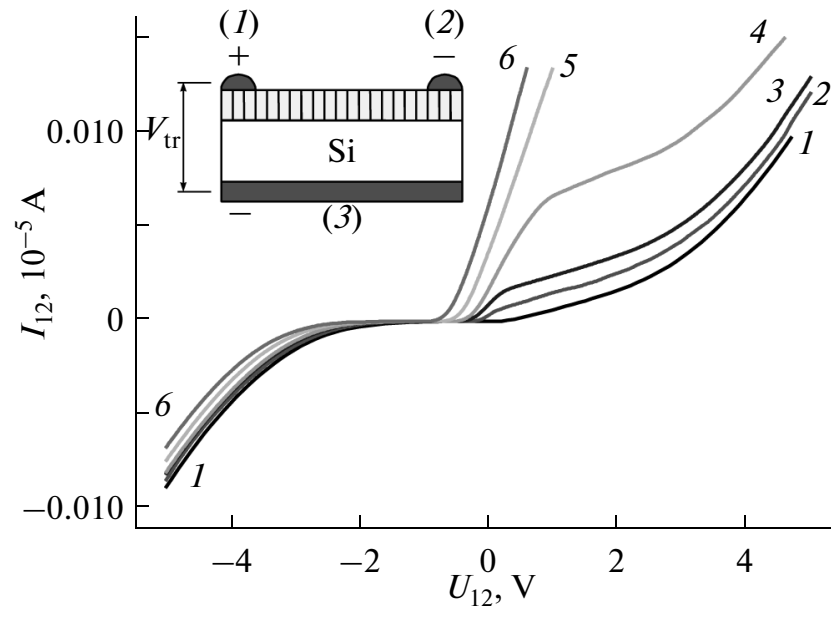

Fig. 3. Voltage-current characteristics $(B=0, T=300 \mathrm{~K})$ at various voltages $V_{\mathrm{tr}}$, V: (1) 0 ; (2) 0.5 ; (3) 0.8 ; (4) 1.0 ; (5) $1.5 ;$ (6) 2.0 .

The results presented in Fig. 2 (obtained at $V_{\mathrm{tr}}=0$ and $B=8 \mathrm{Tl}$ ) indicate that the magnetoresistance $M R_{12}$ for the nanostructures studied depends not only on the temperature but also on the value of the measuring current. In particular, it can be seen that at temperatures below $35 \mathrm{~K}$ the value of $M R_{12}$ substantially increases with a decrease in the measuring current (see curves 1 and 2 in Fig. 2b). Besides, in the absence of transverse voltage, a significant magnetoresistive effect is observed, which achieves $198-548 \%$ at currents of $10<I_{12}<1000 \mathrm{nA}$. In this case, the temperature corresponding to the maximum value of $M R_{12}$, decreases from 25.8 to $21.1 \mathrm{~K}$ through the decrease in current $I_{12}$ (see Insert in Fig. 3a).

At a temperature higher than $35 \mathrm{~K}$, the magnetoresistance $M R_{12}$ decreases with an increase in the temperature; in this case, in dependences $M R_{12}(T)$ a local maximum is observed, which decreases in value, shifting to a region of higher temperatures with an increase in current $I_{12}$. Besides, for both used orientations of vectors $\mathbf{B}$ and $\mathbf{I}$ by currents $I_{12}=10-100 \mathrm{nA}$ the sign of $M R_{12}$ changes from positive to negative at $T>180 \mathrm{~K}$ (Fig. 2b). This can be associated with the anisotropic magnetoresistance in the nickel nanorods (their contribution to the general resistance of the $n-\mathrm{Si} / \mathrm{SiO}_{2} / \mathrm{Ni}$ structure increases with an increase in the temperature due to the rapid decrease in the resistance of the silicon substrate), as was observed in the experiments specially carried out with the use of granular nickel films deposited onto the $n-$ Si substrates at the same regimes $[12,13]$.

The resistance of the structure by current passing through contacts $1-2$ is determined, first and foremost, by a back-biased Schottky barrier, and therefore, it is most probable that the influence of the magnetic field on the value of $R_{12}$ is mostly associated with 
the processes proceeding in the depleted region of this barrier. Taking into account the voltage-current characteristic's shape in Fig. 1, we can assume that in the absence of a magnetic field the processes of impact ionization take place in the depleted region, as a result of which the barrier height decreases due to the capture of minority charge carriers. This is in agreement with the absence of the effect of positive magnetoresistance at high temperatures, when the mobility of charge carriers is small. In agreement with [14-17], the magnetic field hinders the impact's ionization, which results in an increase in the resistance of the $n-\mathrm{Si} / \mathrm{SiO}_{2} / \mathrm{Ni}$ nanostructure.

In the approach proposed in $[10,11]$, it is possible to control the electrophysical properties of template structures by using a silicon substrate as the third electrode and applying an additional (transverse) electric field to the nanostructure. For this purpose, a series of experiments were carried out, in which a certain voltage $V_{\mathrm{tr}}$ was additionally applied (from -2 to $+2 \mathrm{~V}$ ) between the rear and one of the face electrodes (see Insert in Fig. 3). Such a voltage will cause a redistribution of the electric potential in the $n-\mathrm{Si} / \mathrm{SiO}_{2} / \mathrm{Ni}$ nanostructure and, in particular, affect the electrophysical properties of the internal interfaces in $n-\mathrm{Si}$ / $\mathrm{SiO}_{2}$ and $n-\mathrm{Si} / \mathrm{Ni}$ (changing the width of the space's charge regions and the strength of the electric field in them). The results of these experiments are presented in Figs. 3 and 4.

As can be seen from Fig. 3, the dependence of current $I_{12}$ on voltage $V_{12}$ is substantially changed by applying transverse voltage $V_{\text {tr }}$ to the structure. It is evident that a change in voltage $V_{\text {tr }}$ makes it possible to reduce voltage $V_{12}$, which corresponds to the given measuring current $I_{12}$. In this case, even a minor increase in the resistance under the action of the magnetic field, in agreement with Eq. (1), will cause a significant increase in the value of the relative magnetoresistance $M R_{12}$ even by an immaterial change in the absolute value of the electroresistance in the magnetic field.

As the experiments have shown, the application of transverse voltage $V_{\text {tr }}$ substantially changes the temperature dependence of the relative magnetoresistance $M R_{12}$. As can be seen from Fig. 4, when voltage $V_{\text {tr }}$ is applied, the value of $M R_{12}$ substantially increases in the temperature region of $20-30 \mathrm{~K}$, the maximum in the dependence $M R_{12}(T)$ increases and becomes more pronounced, and the effect is enhanced as current $I_{12}$ decreases and voltage $V_{\text {tr }}$ increases. The greatest increase in $M R_{12}$, which achieved $34000 \%$ in the magnetic field of $8 \mathrm{Tl}$, was observed at $V_{\mathrm{tr}}=-2 \mathrm{~V}$ and $I_{12}=$ $100 \mathrm{nA}$ (Fig. 4b). At temperatures $T>35 \mathrm{~K}$ the value of $M R_{12}$ also depends on the value and sign of the bias voltage $V_{\text {tr }}$, although to a lesser degree. In particular, at $V_{\mathrm{tr}}=+2 \mathrm{~V}$ (see curve 2 in Fig. $4 \mathrm{a}$ ) the magnetoresistance becomes negative in the whole region of temper-

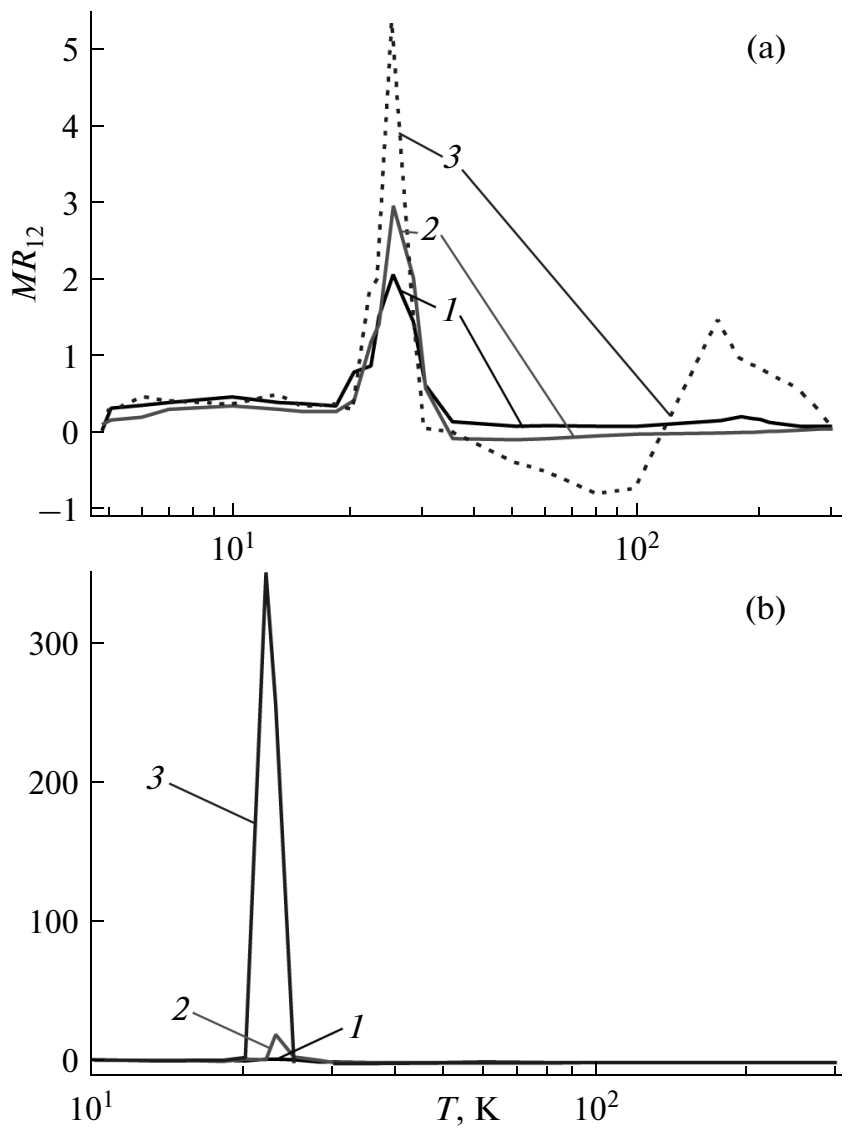

Fig. 4. Temperature dependences of the magnetoresistance $M R_{12}(B=8 \mathrm{Tl})$ obtained by measuring currents of $I_{12}=$ 1000 (a) and 100 (b) $\mathrm{nA}$ at various values of the transverse voltage $V_{\mathrm{tr}}, \mathrm{V}:(1) 0 ;(2)+2 ;(3)-2 \mathrm{~V}$.

atures above $35 \mathrm{~K}$. At the same time, at $V_{\mathrm{tr}}=-2 \mathrm{~V}$ (see curve 3 in Fig. 4a) the value of $M R_{12}$ twice changes the sign by an increase in the temperature: at $T \sim 35 \mathrm{~K}$ from positive to negative, while at $T \sim 120 \mathrm{~K}$, in contrast, from negative to positive. The minimum value of $M R_{12}$ (caused, probably, by the anisotropic magnetoresistance of nickel [18]) is $-86.3 \%$ at $80.0 \mathrm{~K}$, while the maximum high-temperature positive magnetoresistance is $143.6 \%$ at $161.4 \mathrm{~K}$.

\section{CONCLUSIONS}

It is shown that the magnetoresistive properties of $n-\mathrm{Si} / \mathrm{SiO}_{2} / \mathrm{Ni}$ nanostructures containing nanogranular nickel rods in pores in the $\mathrm{SiO}_{2}$ layer substantially differ from the analogous properties for the earlier studied nanogranular Ni films, which were electrodeposited onto the $n-\mathrm{Si}$ plates. From the point of view of the electrophysical properties, the $n-\mathrm{Si} / \mathrm{SiO}_{2} / \mathrm{Ni}$ nanostructures studied are analogous to the system of two Schottky Si/Ni diodes, which are enabled towards each other. 
It is established that $n-\mathrm{Si} / \mathrm{SiO}_{2} / \mathrm{Ni}$ nanostructures possess a substantial positive relative magnetoresistance in the temperature range of $17-27 \mathrm{~K}$, which depends on the current through the sample and can be efficiently controlled by a transverse electric bias voltage achieving $34000 \%$ in the magnetic field of $8 \mathrm{Tl}$. The observed magnetoresistive effect is associated with the influence of the magnetic field on the processes of impact ionization, which lead to an avalanche breakdown of the Schottky barrier.

\section{ACKNOWLEDGMENTS}

The work has been carried out with the support of GPNI "Functional and Engineering Materials, Nanomaterials" of the Republic of Belarus (Task 2.4.08).

\section{REFERENCES}

1. Imry, Y., Nanostructures and Mesoscopic Systems, New York: Academic, 1992, p. 11.

2. Baibich, M.N., Broto, J.M., Fert, A., Nguyen van Dau, F., Petroff, F., Etienne, P., Creuzet, G., Friederich, A., and Chazelas, J., Giant magnetoresistance of (001) Fe/(001)Cr magnetic superlattices, Phys. Rev. Lett., 1988, vol. 61, pp. 2472-2475.

3. Garcia, N., Muñoz, M., and Zhao, Y.-W., Magnetoresistance in excess of $200 \%$ in ballistic Ni nanocontacts at room temperature and 100 Oe, Phys. Rev. Lett., 1999, vol. 82, pp. 2923-2926.

4. Schmidt, G., Gould, C., and Molenkamp, L.W., Spintronics in semiconductor nanostructures, Physica E, 2004, vol. 25, pp. 150-159.

5. Template-based synthesis of nanorod or nanowire arrays, in Springer Handbook of Nanotechnology, Bharat Brushan, Ed., Berlin: Springer, 2007, pp. 161-178.

6. Nanofunctional Materials, Nanostructures, and Novel Devices for Biological and Chemical Detection, Li, C., Zribi, A., Nagahara, L., and Willander, M., Eds., Warrendale, PA: Mater. Res. Soc., 2007.

7. Magnetic Ultrathin Films: Multilayers and Surfaces/Interfaces and Characterization, Jonker, B.T. et al., Eds., MRS Symposia Proceedings no. 313, Warrendale, PA: Mater. Res. Soc., 1990.

8. Fink, D., Petrov, A.V., Hoppe, K., Fahrner, W.R., Papaleo, R.M., Berdinsky, A.S., Chandra,A., Chemseddine, A., Zrineh, A., Biswas, A., Faupel, F., and Chadderton, L.T., Etched ion tracks in silicon oxide and silicon oxynitride as charge injection or extraction channels for novel electronic structures, Nucl. Instrum. Methods Phys. Res. B, 2004, vol 218, pp. 355-361.
9. Streltsov, E.A., Osipovich, N.P., Ivashkevich, L.S., and Lyakhov, A.S., Effect of Cd(II) on electrodeposition of textured PbSe, Electrochim. Acta, 1999, vol. 44, pp. 2645-2652.

10. Fink, D., Sinha, D., Opitz-Coutureau, J., Petrov, A.V., Demyanov, S.E., Fahrner, W.R., Hoppe, K., Fedotov, A.K., Chadderton, L.T., and Berdinsky, A.S., Nanotechnology with ion track-tailored media, in Physics, Chemistry, and Application of Nanostructures, Proceedings of the Nanomeeting-2005, Minsk, Belarus, p. 474.

11. Ivanova, Yu.A., Ivanou, D.K., Fedotov, A.K., Streltsov, E.A., Demyanov, S.E., Petrov, A.V., Kaniukov, E.Yu., and Fink, D., Electrochemical deposition of $\mathrm{Ni}$ and $\mathrm{Cu}$ onto monocrystalline $n$-Si(100) wafers and into nanopores in $\mathrm{Si} / \mathrm{SiO}_{2}$ template, J. Mater. Sci., 2007, vol. 42, pp. 9163-9165.

12. Fedotova, J., Ivanou, D., Ivanova, Yu., Fedotov, A., Mazanik, A., Svito, I., Streltsov, E., Saad, A., Tyutyunnikov, S., Koltunowicz, T.N., Demyanov, S., and Fedotova, V., Magnetoresistance in $n-\mathrm{Si} / \mathrm{SiO}_{2} / \mathrm{Ni}$ nanostructures manufactured by swift heavy ioninduced modification technology, Acta Phys. Polon. A, 2011, vol. 120, pp. 133-135.

13. Fedotova, J., Saad, A., Ivanou, D., Ivanova, Yu., Fedotov, A., Mazanik, A., Svito, I., Streltsov, E., Tyutyunnikov, S., and Koltunowicz, T.N., Gigantic magnetoresistive effect in $n-\mathrm{Si} / \mathrm{SiO}_{2} / \mathrm{Ni}$ nanostructures fabricated by the template-assisted electrochemical deposition, Electric. Rev., 2012, vol. 88, pp. 305308.

14. Delmo, M.P., Yamamoto, Sh., Kasai, Sh., Ono, T., and Kobayashi, K., Large positive magnetoresistive effect in silicon induced by the space-charge effect, Nature, 2009, vol. 457, pp. 1112-1115.

15. Lanyon, H.P.D. and Neal, W.S., Magnetoresistance of silicon diodes reverse biased into breakdown, Phys. Status Solidi A, 1974, vol. 21, pp. 605-616.

16. Lanyon, H.P.D., Magnetoresistance of avalanching semiconductor diodes, Phys. Status Solidi A, 1974, vol. 21, pp. 197-207.

17. Lutsev, L.V., Stognij, A.I., and Novitskii, N.N., Giant magnetoresistance in semiconductor/granular film heterostructures with cobalt nanoparticles, Phys. Rev. $B, 2009$, vol. 80, p. 184423.

18. Fedotova, J., Saad, A., Ivanou, D., Ivanova, Yu., Fedotov, A., Mazanik, A., Svito, I., Streltsov, E., Tyutyunnikov, S., and Koltunowicz, T.N., Magnetotransport in nanostructured Ni films electrodeposited on Si substrate, Electric. Rev., 2012, vol. 88, pp. 90-92.

Translated by R. Litvinov 